\title{
Attenuated frontal and sensory inputs to the basal ganglia in cannabis users
}

\author{
Laura Blanco-Hinojo ${ }^{1}$, Jesus Pujol ${ }^{1,2, *}$, Ben J Harrison ${ }^{3}$, Dídac Macià ${ }^{1}$, Albert \\ Batalla $^{4,5}$, Santiago Nogué ${ }^{6}$, Marta Torrens ${ }^{7,8}$, Magí Farré ${ }^{7,8}$, Joan Deus ${ }^{1,9}$, Rocío \\ Martín-Santos 4,10
}

$=$

1

${ }^{1}$ MRI Research Unit, CRC Mar, Hospital del Mar, Barcelona, Spain

${ }^{2}$ Centro Investigación Biomédica en Red de Salud Mental, CIBERSAM G21, Barcelona, Spain

${ }^{3}$ Melbourne Neuropsychiatry Centre, Department of Psychiatry, The University of Melbourne,

Melbourne, Australia

${ }^{4}$ Department of Psychiatry and Psychology, Hospital Clínic, Institut d'Investigació Biomédica August

Pi I Sunyer (IDIBAPS), CIBERSAM G25, Barcelona, Spain

${ }^{5} \mathrm{Nijmegen} \mathrm{Institute} \mathrm{for} \mathrm{Scientist-Practitioners} \mathrm{in} \mathrm{Addiction,} 6500 \mathrm{HE}$, Nijmegen, The Netherlands

${ }^{6}$ Section of Clinical Toxicology, Emergency Service, Hospital Clínic, IDIBAPS, University of

Barcelona, Barcelona, Spain

${ }^{7}$ Human Pharmacology and Clinical Neurosciences, Institute of Neuropsychiatry and Addiction,

Hospital del Mar Medical Research Institute, Barcelona, Spain

${ }^{8}$ School of Medicine, Autonomous University of Barcelona, Red de Trastornos Adictivos (RETIC),

Barcelona, Spain

${ }^{9}$ Department of Clinical and Health Psychology, Autonomous University of Barcelona, Barcelona,

Spain

${ }^{10}$ Department of Psychiatry and Clinical Psychobiology, University of Barcelona, Barcelona, Spain

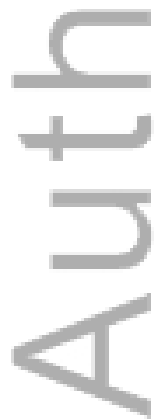

\section{*Corresponding author:}

Dr. Jesus Pujol

Department of Magnetic Resonance, CRC-Mar, Hospital del Mar

This is the author manuscript accepted for publication and has undergone full peer review but has not been through the copyediting, typesetting, pagination and proofreading process, which may lead to differences between this version and the Version of Record. Please cite this article as doi: $10.1111 / \mathrm{adb} .12370$

This article is protected by copyright. All rights reserved. 
Passeig Marítim 25-29 08003 Barcelona, Spain, Fax: + 34932212181 E-mail:.jpujol@crccorp.es

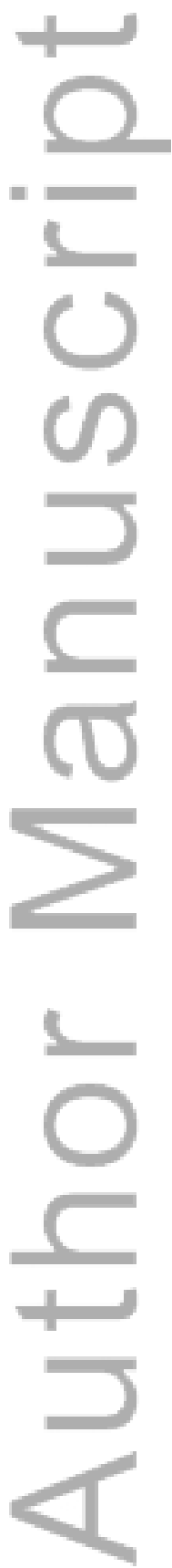

This article is protected by copyright. All rights reserved. 


\begin{abstract}
Heavy cannabis use is associated with reduced motivation. The basal ganglia, central in the motivation system, have the brain's highest cannabinoid receptor density. The frontal lobe is functionally coupled to the basal ganglia via segregated frontalsubcortical circuits conveying information from internal, self-generated activity. The basal ganglia, however, receive additional influence from the sensory system to further modulate purposeful behaviors according to the context. We postulated that cannabis use would impact functional connectivity between the basal ganglia and both internal (frontal cortex) and external (sensory cortices) sources of influence. Restingstate functional connectivity was measured in 28 chronic cannabis users and 29 controls. Selected behavioral tests included reaction time, verbal fluency and exposition to affective pictures. Assessments were repeated after one month of abstinence. Cannabis exposure was associated with (i) attenuation of the positive correlation between the striatum and areas pertaining to the "limbic" frontal-basal ganglia circuit, and (ii) attenuation of the negative correlation between the striatum and the fusiform gyrus, which is critical in recognizing significant visual features. Connectivity alterations were associated with lower arousal in response to affective pictures. Functional connectivity changes had a tendency to normalize after abstinence. The results overall indicate that frontal and sensory inputs to the basal ganglia are attenuated after chronic exposure to cannabis. This effect is consistent with the common behavioral consequences of chronic cannabis use concerning diminished responsiveness to both internal and external motivation signals. Such an
\end{abstract}


impairment of the fine-tuning in the motivation system notably reverts after abstinence.

Key words: arousal, addiction, basal ganglia, cannabis use, fMRI, motivation

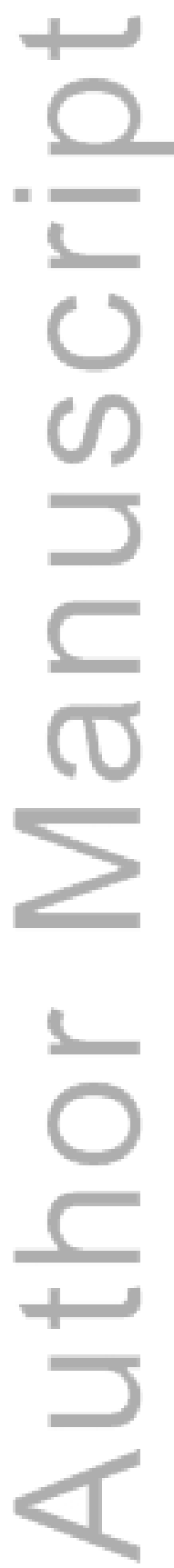

This article is protected by copyright. All rights reserved. 


\section{Introduction}

Chronic cannabis use may significantly compromise both perceptual and executive functioning. Reported alterations include less efficient performance on tasks of visuomotor integration, time estimation, motor control and decisionmaking (King et al, 2011; Skosnik et al, 2014; Solowij et al, 2002; Wesley et al, 2011). Significant impairments in the emotional and motivational domains have also been identified, including poorer facial emotion recognition (Bayrakci et al, 2014; Hindocha et al, 2014), and reduced sensitivity to experimentally induced negative emotional states (Somaini et al, 2012) and reward processing (Martin-Soelch et al, 2009). This broad profile of behavioral changes is compatible with an effect of cannabis on brain structures critical to the integration of multiple-source information.

The basal ganglia complex is a firm candidate to mediate a variety of cannabis effects due to both their high density of cannabinoid receptors (actually the highest density in the brain) (Herkenham et al, 1990) and their central location in the modulation of the entire span of brain responses. In the role of modulating motor, cognitive and emotional responses, the basal ganglia integrate information from different sources. As part of the frontal-basal ganglia circuitry, the striatum (caudate and putamen) receives excitatory afferents from functionally specialized motor, associative and limbic regions of the frontal cortex (Alexander et al, 1986; Haber, 2003). This frontal cortical input is classically conceptualized as the primary influence on basal ganglia conveying information from internal, self-generated mental activity. However, relevant sources of external, sensory information also target the basal ganglia. Individual striatal regions receive some auditory, visual and 
somatosensory inputs via thalamostriatal projections (Marchand, 2010; Shulz et al, 2009; Webster, 1975; Yin and Knowlton, 2006), as well as through direct corticostriatal projections arising from sensory and association areas (e.g., inferior temporal cortex, superior temporal gyrus) (Brown et al, 1997; Connor and Abbs, 1990; Seger, 2013; Wilson, 2014). Indirect sensory inputs involve amygdalar and hippocampal projections (Alexander et al, 1986; Marchand, 2010). Also, the midbrain dopaminergic system, which has a fundamental role in modulating striatal activity, is highly sensitive to salient and arousing sensory stimulation (Da Cunha et al, 2012; Horvitz, 2000). A dual cortico-basal ganglia circuitry is thus involved in constructing appropriate purposeful behaviors according to both internal motivation and external constraints.

Previous neuroimaging studies have identified functional changes in the basal ganglia related to cannabis use (Batalla et al, 2013). Abnormal neural responses in the striatum, as measured with fMRI, have been consistently reported (Batalla et al, 2013; Jager et al, 2013; Nestor et al, 2010; van Hell et al, 2010;). In addition to task-related experiments, MRI analysis of spontaneous brain activity allows the integrity of relevant functional networks to be tested on the basis of region activity synchronization - typically defined as "functional connectivity" (Fox and Raichle, 2007). A recent study using multi-voxel pattern analysis to classify cannabis users from controls revealed that functional connectivity alterations may indeed be present under resting state conditions, with key discriminating areas being the frontal cortex and fusiform gyrus (Cheng et al, 2014). We have also recently reported that chronic 
cannabis use alters functional connectivity between higher-order brain networks relevant to self-awareness (Pujol et al, 2014a).

Functional connectivity studies in healthy subjects have consistently shown that the striatum is positively connected with frontal cortical areas, and negatively connected with sensory cortices and the hippocampus (Barnes et al, 2010; Di Martino et al, 2008; Harrison et al, 2009). Although the positively connected frontal circuits have attracted more attention, the negative functional coupling between sensory cortices and basal ganglia also is very robust (Di Martino et al, 2008). Such a functional relationship between the sensory cortices and the basal ganglia is highly dynamic. The negative correlation (anticorrelation) observed under resting state conditions, may be seen as a positive correlation (co-activation) during tasks involving meaningful visual stimulation (Anderson et al, 2014; Butler et al, 2007; Seger, 2013).

Presynaptically located, cannabinoid receptors are ideally positioned to modulate the balance of excitation and inhibition (McLaughlin et al, 2014). Excessive stimulation of cannabinoid receptors in chronic users could affect the function of the basal ganglia and attenuate the influence of their afferent inputs. Our hypothesis is that this effect will impact on the functional connectivity of the basal ganglia with both internal (frontal cortex) and external (sensory cortices) sources of influence. In the present study, we used resting-state fMRI to examine cannabis effects on basal ganglia functional connectivity in early-onset chronic cannabis users without comorbid psychiatric disorders. A selected cognitive assessment was conducted including reaction time to broadly explore motor system readiness, a verbal fluency 
task to assess the capacity to internally generate cognitive activity and the exposition to affective pictures to assess responsiveness to external stimulation. Whole-brain correlation mapping served to determine the extent to which potential connectivity changes were related to cannabis users' performance in these tests. Imaging data was initially acquired during active cannabis use, but in the unintoxicated state (i.e., explored at least 12 hours after the last cannabis use). The assessment was repeated = after one month of controlled abstinence with the goal of addressing potentially enduring alterations."

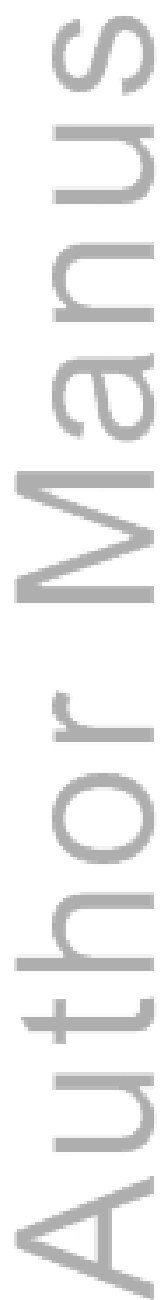




\section{Materials and methods}

\subsection{Participants}

Twenty-eight chronic cannabis user men (mean \pm SD age, $21 \pm 2$ years) were assessed and compared with a control group of 29 non-user men (age, $22 \pm 3$ years, ns). Participants were recruited via a webpage and distribution of flyers and ads. To evaluate study eligibility, a comprehensive telephone screening was carried out. When eligible, participants were assessed using a detailed medical history, physical examination, a structured psychiatric interview for substance users (PRISM-DSM-IV; Torrens et al, 2004), blood biochemical analyses and urine toxicology analyses (immunometric assay kits, Instant-View; ASD Inc., Poway, California).

Inclusion to the cannabis group required participants to be male, aged between 18 and 30 years, with at least 10 years of education (mean \pm SD, $14 \pm 2$ years), cannabis use onset before age 16, cannabis consumption (smoking) more than 14 times a week at the time of selection and during at least 2 years prior to study entry, positive urine test for cannabinoids and negative for opiates, cocaine, amphetamines and benzodiazepines. Exclusion criteria included: Diagnostic and Statistical Manual for Mental Disorders-Fourth Edition (DSM-IV; American Psychiatric Association, 2000) Axis I disorder, other than cannabis dependence disorder, relevant medical or neurological disorders, learning disabilities, use of psychoactive medications, previous lifetime use of any other recreational drug of more than 5 occasions lifetime (except alcohol and nicotine), lifetime criteria for alcohol abuse or dependence and relevant current alcohol consumption. As a consequence of a strict sample selection, 
current alcohol intake was very low in both study groups, showing a mean \pm SD of $5.3 \pm 4$ units a week in users and $3.1 \pm 2.6$ units a week in control subjects. On average, cannabis users smoked a mean \pm SD of $5.9 \pm 5.2$ cigarettes a day and control subjects, $2.4 \pm 5.9$ cigarettes a day. Only three participants (two users and one control subject) smoked more than 10 cigarettes per day. All participants were right-handed. (

$=$

Control subjects were also required to be male, aged between 18 and 30 years, with at least 10 years of education $(15 \pm 1$ years), with less than 15 lifetime experiences with cannabis (none in the past month) and negative urine drug screen. Exclusion criteria were identical to the cannabis group. Cannabis users and control subjects showed a mean difference of 1 year in education $(\mathrm{t}=2.2, \mathrm{p}=0.032)$. Therefore, study analyses were performed controlling for this variable when appropriate.

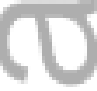

Participants were required to refrain from cigarette smoking and caffeine 6 hours, and alcohol and cannabis $12 \mathrm{~h}$ before the scanning session. All of them reported full accomplishment of these conditions for each substance. The study consisted of two fMRI assessments. The second fMRI session was carried out in all available participants after a period of 28 days of controlled cannabis abstinence. During this abstinence period, use of drugs of abuse, including cannabis, was checked with urine weekly drug screenings scheduled on days 7, 14, 21 and 28. Subjects were expected to exhibit negative urine tests for all drugs of abuse but cannabinoids and were monitored for levels of the metabolite 11-nor-9-carboxy-delta9-

tetrahydrocannabinol (THCCOOH). All cannabis users included in the study reported

This article is protected by copyright. All rights reserved. 
abstaining from cannabis use through the follow-up. Quantitative measures in urine

were performed by immunoassay with values $<50 \mathrm{ng} / \mathrm{mL}$ classified as negative.

Cannabis users were considered abstinent provided a decrease of creatinine-corrected

baseline urinary THCCOOH concentrations.

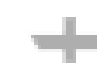

⿶

Written informed consent was obtained from all participants. The study was

approved by the local ethics committee (CEIC-IMAS, CEIC-Hospital Clínic,

Barcelona) and was in compliance with the Declaration of Helsinki.

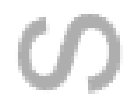

\subsection{Behavioral assessment}

Prior to the scanning session, participants underwent three brief behavioral tests known to be sensitive to striatal dysfunction (Thames et al, 2012) including a computerized version of the Motor Screening Test (MOT, included in the CANTAB neuropsychological battery; Automated CCNT, 2006), a verbal fluency test (Benton et $a l, 1983)$ and a picture-viewing task (using the International Affective Picture System, IAPS; Lang et al, 1995). The MOT is a simple pointing task and was conducted to assess subjects' general motor system readiness. Participants were instructed to point on a flashing cross as soon as it appeared in the computer screen. For the verbal fluency task, participants were requested to produce as many words in $1 \mathrm{~min}$ as possible that belonged to a specific semantic category, namely animals. A set of standardized IAPS color photographs was used as emotionally evocative visual stimuli. Participants were asked to rate each picture on two dimensions (i.e., valence and arousal) based on the intensity of the emotion that the picture elicited using a 9- 
point scale (1 = "very negative" or "low intensity/arousal"; 9 = "very positive" or "high intensity").

The primary outcome variables obtained from the different probes were: response latency and accuracy in the MOT, total number of correctly generated words in $60 \mathrm{~s}$ in the verbal fluency test and mean valence and arousal scores in the IAPS.

\subsection{Image acquisition and preprocessing}

MRI acquisition. Images were acquired with a 1.5 Tesla Signa Excite system (General Electric, Milwaukee, WI, USA) equipped with an eight-channel phasedarray head coil and single-shot echoplanar imaging (EPI) software. The functional sequence consisted of gradient recalled acquisition in the steady-state (time of repetition [TR], $2000 \mathrm{~ms}$; time of echo [TE], $50 \mathrm{~ms}$; pulse angle, 90 $90^{\circ}$ within a field of view of $24 \mathrm{~cm}$, with a 64 x 64-pixel matrix, and slice thickness of $4 \mathrm{~mm}$ (inter-slice gap, $1.5 \mathrm{~mm})$. Twenty-two interleaved slices were prescribed parallel to the anteriorposterior commissure line covering the whole brain. A 6-min continuous resting-state scan was acquired for each participant, generating 180 whole brain EPI volumes. The first four (additional) images in each run were discarded to allow magnetization to reach equilibrium. For this sequence, participants were instructed to relax, stay awake and to lie still without moving, while keeping their eyes closed throughout.

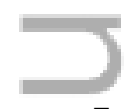

Image pre-processing. Imaging data were processed using the Statistical Parametric Mapping software (SPM8; The Wellcome Department of Imaging Neuroscience, http://www.fil.ion.ucl.ac.uk/spm/), running on Matlab version 2011b 
(The Mathworks Inc., Natick, Mass). Preprocessing involved: (i) conventional rigid body realignment procedures to correct for head movement, (ii) spatial normalization to the standard SPM-EPI template and reslice of the functional images to $2 \mathrm{~mm}$ isotropic resolution in Montreal Neurological Institute (MNI) space, and (iii)

smoothing using a Gaussian filter (full-width half-maximum, $8 \mathrm{~mm}$ ). A high-pass filter set at 128 seconds was used to remove low-frequency drifts of less than approximately $0.008 \mathrm{~Hz}$. All image sequences were inspected for potential acquisition and normalization artifacts. One cannabis user was excluded from an original sample of 29 subjects due to non-optimal data acquisition.

Head motion measurements. Motion was quantified using realignment parameters obtained during image preprocessing, which included 3 translation and 3 rotation estimates. Average inter-frame motion measurements (head position variations of each brain volume as compared to the previous volume) were used to capture head motion across the 6-minute scan. For each subject, a motion summary measurement that combined translations and rotations was computed in $\mathrm{mm}$ by adapting the formula of Van Dijk et al. (2012). We compared both study groups as for potential differences in movement for translations (mean $\pm \mathrm{SD}, 0.053 \pm 0.02$ controls, $0.042 \pm 0.02$ cannabis users), rotations (mean $\pm \mathrm{SD}, 0.029 \pm 0.01$ controls, $0.027 \pm 0.01$ cannabis users), and mean inter-frame motion (mean $\pm \mathrm{SD}, 0.041 \pm 0.01$ controls, $0.035 \pm 0.01$ cannabis users) and found no significant differences in any parameter. 


\subsection{Seed-based functional connectivity analyses}

To assess potential differences in the pattern of functional connectivity of specific striatal subdivisions, we performed a detailed seed-based cross-correlation analysis of subjects' resting-state imaging sequences. Functional connectivity seed maps of the regions of interest were generated adapting the procedures detailed in Harrison et al. (2009) after Di Martino et al. (2008). Briefly, for each location, seeds were defined using the MarsBar region-of-interest toolbox (Brett et al, 2002) as 3.5 $\mathrm{mm}$ radial spheres (sampling approximately 25 voxels in $2 \mathrm{~mm}$ isotropic resolution) with a minimum Euclidean distance requirement of $8 \mathrm{~mm}$ between any two regions, centered at the following bilateral MNI coordinates: a) dorsal caudate, $x=13, y=15$, $\mathrm{z}=9$; b) dorsal putamen, $\mathrm{x}=28, \mathrm{y}=1, \mathrm{z}=3$; c) ventral caudate, corresponding approximately to the nucleus accumbens, $x=9, y=9, z=-8$; and d) ventral putamen, $x=20, y=12, z=-3$. Signal values for the seeds ( 8 in total) were calculated as the average signal of the voxels included in the seed at each time point.

To generate the seed maps, the signal time course of the selected seed region was used as a regressor to be correlated with the signal time course of every voxel in the brain, and the obtained voxel-wise regression coefficients were represented as first-level SPM contrast images. This process was performed for each subject and seed separately. To remove potential sources of physiological noise, we derived estimates of brain tissue signal fluctuations to be included as confounding variables in the multiple regression SPM models together with the variable of interest. Using normalized standard masks in MNI space from SPM, white matter, CSF and global 
First-level contrast images, estimated for each participant, were then included in second-level (group) random-effects analyses. One-sample t-statistic maps were calculated to obtain functional connectivity maps for each group, and two-sample ttests were performed to map between-group differences. SPM linear regression was used to estimate the correlation between (self-reported) cannabis use measurements (years of cannabis use, average joints per time and cannabinoid metabolites as independent regressors) and voxel-wise functional connectivity in the obtained maps in the cannabis users group. Voxel-wise correlation analyses were also performed in SPM between behavioral measurements (i.e., IAPS-related arousal) and seed functional connectivity in both groups. In order to address the difference in educational level between groups, the seeds and correlation maps were re-estimated after covarying for subjects' years of education.

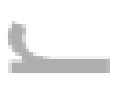

Thresholding criteria. To identify functional connectivity networks in onesample analyses, between-group differences and correlation analyses between behavioral ratings and connectivity measurements, results were considered significant with clusters of $1.032 \mathrm{ml}(>129$ voxels $)$ at a height threshold of $\mathrm{p}<0.005$, which satisfied the family-wise error (FWE) rate correction of $\mathrm{P}_{\mathrm{FWE}}<0.05$ according to recent Monte Carlo simulations (Pujol et al, 2014a; 2014b). 


\subsection{Statistical analysis of behavioral data.}

Student t-test was used to compare demographic and behavioral variables between groups. Repeated measures ANOVAs were conducted to examine possible differences between behavioral ratings in the baseline and post-abstinence acquisitions, and ANCOVA was used instead when covariates were included in the comparison.

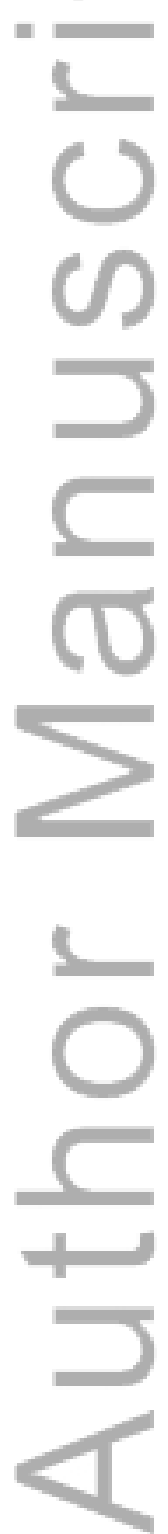

This article is protected by copyright. All rights reserved. 


\section{Results}

\subsection{Behavioral assessment}

Estimates of cannabis use and summary statistics for the behavioral tests are shown in Table 1. At baseline, cannabis users and control subjects only differed in their arousal ratings on the IAPS test. On average, cannabis users rated the pictures $6.1 \%(95 \%$ CI $[0.7,11.4])$ less arousing $(\mathrm{p}=0.026)$ than control participants. After controlling for the effect of education, group differences in arousal ratings remained significant $[\mathrm{F}=5.46$ and $\mathrm{p}=0.023]$.

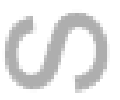

After 28 days of cannabis abstinence, we found no significant between-group differences in any of the main performance indices on the tasks. Though cannabis users again tended to evaluate images from the IAPS as less arousing than healthy controls, the difference was not statistically significant.

\subsection{Functional connectivity}

Within-group maps. Overall, both groups exhibited significant and robust patterns of striatal functional connectivity. Positive correlations were found between the striatal seed regions and a distributed set of cortical areas involving frontal and parietal cortex and subcortical structures involving the whole bilateral striatum, globus pallidus, thalamus, subthalamic region and upper mesencephalon (Supplementary Material, Tables S1-S4 and Figures S1 and S2). The caudate nucleus was more densely connected to the prefrontal cortex and rostral anterior cingulate cortex (ACC), whereas the putamen was more connected to motor regions and supplementary motor 
area (SMA). Negative functional connectivity with the striatal seeds mostly involved superior parietal regions, occipital cortices - including primary visual areas, the lingual gyrus and the fusiform gyrus-, portions of the temporal cortex, and superior parts of the cerebellum (Supplementary Material, Tables S1-S4 and Figures S1 and S2). No substantial hemispheric differences were noted for any of the connectivity maps.

피 3

Between-group differences. Cannabis users exhibited abnormal functional connectivity between the striatum and cortical areas in both the positively correlated and negatively correlated (anticorrelated) systems with notably overlapping results across the different seed maps (Figure 1 and Tables S1-S4). As the most consistent finding, cannabis users showed a significant reduction of the positive correlation between several striatal seed regions and the ACC/medial frontal cortex, as well as a significant reduction of the negative correlation between several striatal regions and the fusiform gyrus bilaterally (Figure 1).

$\$$

To extend the analysis and explore the reciprocity of functional connectivity alterations described above, additional maps were generated placing seeds at peak between-group differences (ACC at MNI coordinates: $\mathrm{x}=8, \mathrm{y}=26, \mathrm{z}=28$, and fusiform gyri at right $\mathrm{x}=33, \mathrm{y}=-51, \mathrm{z}=-21$, and left, $\mathrm{x}=-36, \mathrm{y}=-44, \mathrm{z}=-20$ ). Figure $\mathrm{S} 3$ in Supplementary Material shows the within-group functional connectivity maps from this analysis and Figure 2 and Table S5 show between-group differences. Consistent with the study's primary results, cannabis users showed a significant reduction of the 
positive correlation between the ACC and the basal ganglia, and a significant reduction of the negative correlation between both fusiform gyri and the basal ganglia. These results are highly demonstrative of the association of cannabis use with attenuated functional coupling of the basal ganglia with converging frontal and

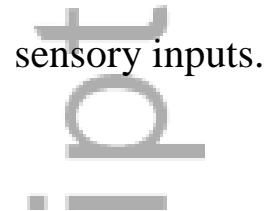

\subsection{Correlation analyses}

\section{Correlation of functional connectivity changes with measurements of cannabis}

use. Within the cannabis user group, voxel-wise regression analysis revealed a significant negative correlation between years of cannabis use and functional connectivity measurements in the $\mathrm{ACC} /$ medial frontal cortex in both the (right) dorsal and (left) ventral caudate seed maps. Consistently, a significant positive correlation was also found between cannabinoid metabolites present in urine and functional connectivity in the left fusiform gyrus in the (right) ventral putamen seed map. Figure 3 shows scatter plots illustrating these correlations.

Correlation with behavioral ratings. This analysis was limited to the behavioral variable showing significant between-group differences (i.e., IAPS mean arousal ratings). As to the primary basal ganglia functional connectivity maps, cannabis users versus controls showed stronger positive correlation between arousal ratings and functional connectivity between the caudate nucleus and medial prefrontal cortex, posterior cingulate cortex, and bilateral angular gyri, which are major constituents of the default mode network. Cannabis users also showed stronger negative correlation 
between arousal ratings and functional connectivity between the caudate nucleus and the sensorimotor cortex bilaterally (Figure 4 and Table S6), which is in the striatum negative correlation map.

As to the ACC and fusiform gyrus functional connectivity maps, arousal ratings interestingly correlated with functional connectivity measurements in the basal ganglia itself. Specifically, cannabis users versus controls showed stronger positive correlation between arousal ratings and functional connectivity between the ACC and the basal ganglia, and stronger negative correlation between arousal ratings and functional connectivity between the right fusiform gyrus and basal ganglia (Figure 4, Table S6). Note the resemblance between the pattern of correlations and the pattern of between-group differences in the ACC and fusiform seed maps (Figure 2).

\subsection{Long-term cannabis use effect on functional connectivity}

After one month of supervised abstinence, no significant between-group differences were observed in the regions showing changes at baseline. Findings above threshold were only identified in orbitofrontal areas with no group effect during cannabis use. Nevertheless, when a more lenient threshold was employed $(p<0.01$ uncorrected, 129 voxels), between-group differences persisted for connectivity decreases in the (left) dorsal and (right) ventral caudate seed maps involving the medial frontal cortex/ACC $(\mathrm{MNI} x=12, \mathrm{y}=-6, \mathrm{z}=56, \mathrm{~T}=2.8$ and $\mathrm{x}=-14, \mathrm{y}=26, \mathrm{z}=24$, $\mathrm{T}=3.1$ ), suggesting partial normalization of functional changes in the cannabis user group. 


\section{Discussion}

Chronic cannabis use was associated with abnormal functional connectivity between the striatum and cortical areas, with respect to both the positively correlated (frontal cortex) and anticorrelated (sensory cortex) systems. The areas showing the most consistent positive correlation reduction involved the ACC and adjacent medial prefrontal cortex. The most consistent anticorrelation reduction involved the fusiform gyrus. Relevantly, these observed functional connectivity alterations showed a tendency to normalize after 28 days of controlled abstinence.

The ACC is the primary cortical component of the basal ganglia "limbic loop" (Cummings, 1993) and receives direct input from dopaminergic brainstem neurons and amygdala, signaling the arousal/drive state of the organism (Paus, 2001). This circuit is generally conceptualized as an "auto-activation" platform (Laplane and Dubois, 2001) involved in the integration of emotional/motivational information with purposeful (i.e., goal-directed) behavioral responses (Habib, 2004). Disruption of this system as a result of neurological lesions is well known to be associated with diminished drive (e.g., apathy, loss of interest, absence of spontaneous actions, psychomotor slowing and blunted affect) (Cummings, 1993; Habib, 2004).

In this context, our finding of attenuated functional connectivity within the striatal-ACC circuit is consistent with the notion that heavy cannabis use may significantly affect motivation (Filbey et al, 2013; Volkow et al, 2014). Lowered alertness (Wadsworth et al, 2006), anhedonia and reduced reactivity to reward (Dorard et al, 2008; Martin-Soelch et al, 2009) are frequent observations in chronic cannabis users. Indeed, expressions such as feeling tired, fatigued, low in energy and 
unmotivated are common in self-reports of the adverse consequences of heavy cannabis use (Patton et al, 2002; Reilly et al, 1998). Neuroimaging studies have certainly provided converging evidence of dysfunctional ACC and medial prefrontal cortex in chronic cannabis users in tasks involving, for example, decision-making (Wesley et al, 2011), error-awareness (Hester et al, 2009), response inhibition and responses to negative reward (Eldreth et al, 2004; Gruber et al, 2009).

Our other central finding in chronic cannabis users involved a significant attenuation of the typically observed negative functional coupling between the striatum and the fusiform gyrus. The fusiform gyrus is closely functionally linked to the hippocampus and extended amygdala complex, as part of the ventral visual processing stream, to jointly mediate individual's reactions according to the significance of external stimuli (Haxby et al, 2002). Behavioral studies in heavy cannabis users have reported impairments in visuo-perceptual domains with notable fusiform gyrus participation, including slower and less accurate emotional face recognition (Bayrakci et al, 2014; Hindocha et al, 2014; Platt et al, 2010) and lower arousal in response to affective pictures (Somaini et al, 2012).

In our study, lower arousal in response to affective pictures was significantly associated with attenuated connectivity of the fusiform gyrus and ACC with the striatum supporting the notion that diminished responsiveness in cannabis users may, at least in part, be mediated by altered modulation of the basal ganglia system. Lower arousal was also associated with enhanced connectivity between the striatum and the default mode network. This association could reflect an attentional bias to self- 
referential processes in detriment to the individual's readiness to respond to external stimuli in cannabis users (Pujol et al, 2014a).

After 28 days of cannabis discontinuation, abnormal reductions in basal ganglia functional connectivity were still observed in the cannabis user group, albeit mostly attenuated. Similarly, there was a general tendency for the magnitude of observed differences in arousal ratings to be reduced in the follow-up. These findings

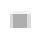
suggest that functional changes associated with active cannabis use have a tendency to recover with abstinence. However, this does not mean that the brain, and particularly the reward system, fully normalizes after cleaning the drug. Indeed, longterm-studies in substance users have shown that the consequences of cumulative exposure to a substance can continue to affect motivational processes and cognitive funetioning well after the cessation of use. Heavy cannabis use has been associated with altered motivational processes (e.g. strong motivations towards drug use) involving cue-induced craving, attentional biases and approach tendencies (Cousijn, 2015), thought to play an important role in continued substance use and relapse. The potentially abnormal functioning of the reward system in chronic cannabis users during the abstinent state could be further investigated with new functional connectivity approaches and, mostly, by testing its responsiveness in cue-elicited craying experiments using fMRI (Parvaz et al, 2011).

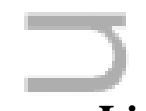

Limitations. Alcohol and nicotine use frequently co-occur with cannabis. Our recruitment criteria addressed this potential limitation and only participants with low alcohol and cigarette use were included. However, all cannabis users in our study 
smoked cannabis combined with tobacco (the most common form of cannabis use in

Spain). Therefore, although cannabis may arguably be the major responsible for the

observed alterations, we cannot exclude potential confounding effect of nicotine.

Also, correlational studies may not be appropriate for making direct statements

regarding the causal role of cannabis. Nonetheless, the observed association between

cannabis use variables and functional connectivity changes, in addition to observed

effects of abstinence, suggest such relationship may exist. Additionally, our study

does not allow distinguishing between direct drug actions on cannabis receptors and

long-lasting effects on brain functional connectivity (shaping) in the involved

systems.Further studies assessing cannabis dose-functional connectivity relationships

may be important for elucidating this issue. Finally, a 6 min resting-state fMRI may

be eonsidered a relatively short period.

To the extent that functional connectivity relates to neural activity integration (Leopold and Maier, 2013), the results of the current study indicate that frontal and sensory inputs to the basal ganglia are significantly attenuated in chronic cannabis users. This effect is consistent with the common behavioral consequences of chronic cannabis exposure concerning blunted responsiveness to both internal and external motivation signals. Importantly, abstinence appears to partly reverse the effects of cannabis on the fine-tuning of the brain's motivation system. These relationships should now be explored in the context of vulnerability or proneness to mental illness, particularly, to an elevated risk of psychosis.

This article is protected by copyright. All rights reserved. 


\section{Acknowledgements}

This study was supported in part with Spanish grants: Plan Nacional sobre Drogas, Ministerio de Sanidad y Consumo, PNSD 2006/101 and PNSD 2011/050 (R. MartínSantos). The Agency of University and Research Funding Management of the Catalonia Government participated in the context of Research Groups SGR2009/1435 and SGR2014/1135 (R. Martín-Santos), and SGR2014/1673 (J. Deus). Ms. BlancoHinojo was supported by the PFIS grant FI10/00387 from the Carlos III Health Institute. Dr. Harrison is supported by a National Health and Medical Research Council of Australia (NHMRC) Clinical Career Development Award (I.D. 628509).

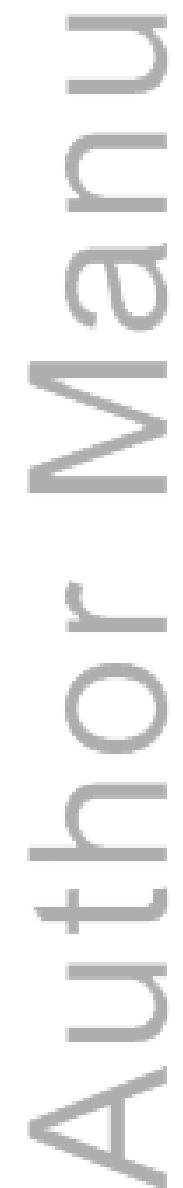

This article is protected by copyright. All rights reserved. 


\section{Authors' contribution}

RMS and JP were responsible for the study design. AB, MF, SN and MT conducted the clinical characterization and selection of participants. LBH, JD and JP contributed to design the fMRI protocols, performed the neuroimaging, and analyzed fMRI results. DM developed fMRI analysis tools and assisted with data analysis. LBH drafted the manuscript. JP, BJH, DM, JD, and RMS contributed to interpretation of $=$ findings and provided critical revision of the manuscript for important intellectual content. All authors have revised and approved the manuscript for publication.

\section{Conflicts of interest}

The authors declare no conflict of interest.

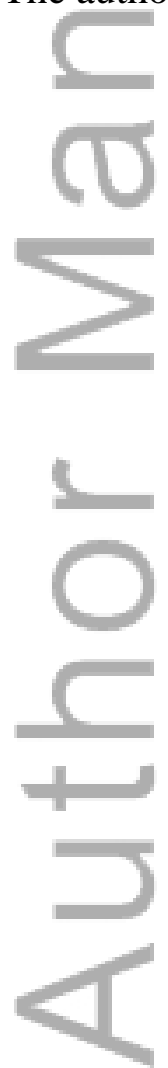

This article is protected by copyright. All rights reserved. 


\section{References}

Alexander GE, DeLong MR, Strick PL (1986). Parallel organization of functionally segregated circuits linking basal ganglia and cortex. Annu Rev Neurosci 9: $357-381$.

American Psychiatric Association (1994). Diagnostic and Statistical Manual of

$=$ Mental Disorders, 4 th ed, text revision. Washington, DC: American

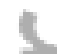
Psychiatric Press.

Anderson BA, Laurent PA, Yantis S (2014). Value-driven attentional priority signals in human basal ganglia and visual cortex. Brain Res 1587: 88-96.

Automated CCNT (2006) Cambridge, England: Cambridge Cognition Limited.

Barnes KA, Cohen AL, Power JD, Nelson SM, Dosenbach YB, Miezin FM et al (2010). Identifying basal ganglia divisions in individuals using resting-state functional connectivity MRI. Front Syst Neurosci 10: 4-18.

Batalla A, Bhattacharyya S, Yucel M, Fusar-Poli P, Crippa JA. Nogue S et al (2013). Structural and functional imaging studies in chronic cannabis 1 users: a systematic review of adolescent and adult findings. PLoS One 8: e55821.

Bayrakci A, Sert E, Zorlu N, Erol A, Sariçiçek A, Mete L (2014). Facial emotion $+\quad$ recognition deficits in abstinent cannabis dependent patients. Compr Psychiatry 58: 160-164.

Benton AL, Hamsher K, Sivan A (1983). Multilingual Aphasia Examination. Iowa City: AJA Associates. 
Brett MA, Anton JL, Valabregue R, Poline JB (2002). Region of interest analysis using an SPM toolbox. Neuroimage 16: 497 (abstract).

Brown LL, Schneider JS, Lidsky I (1997). Sensory and cognitive functions of the basal ganglia. Curr Opin Neurobiol 7: 157-163.

Butler T, Pan H, Tuescher O, Engelien A, Goldstein M, Epstein J et al (2007). Human fear-related motor neurocircuitry. Neuroscience 150: 1-7.

Cheng H, Skosnik PD, Bruce BJ, Brumbaugh MS, Vollmer JM, Fridberg DJ et al (2014). Resting state functional magnetic resonance imaging reveals distinct brain activity in heavy cannabis users - a multi-voxel pattern analysis. J Psychopharmacol 28: 1030-1040.

Connor NP, Abbs J (1990). Sensorimotor contributions of the basal ganglia: Recent advances. Phys Ther 70: 864-872.

Cousijn (2015). Embracing comorbidity: a way toward understanding the role of motivational and control processes in cannabis use disorders. Front Psychol 6:
677.

Cummings JL (1993). Frontal-subcortical circuits and human behavior. Arch Neurol 50: $873-880$.

Da Cunha C, Gómez-A A, Blaha CD (2012). The role of the basal ganglia in motivated behavior. Rev Neurosci 23: 747-767.

Di Martino A, Scheres A, Margulies DS, Kelly AM, Uddin LQ, Shehzad Z et al (2008). Functional connectivity of human striatum: a resting state FMRI study. Cereb Cortex 18: 2735-2747.

This article is protected by copyright. All rights reserved. 
Dorard G, Berthoz S, Phan O, Corcos M, Bungener C (2008). Affect dysregulation in cannabis abusers: a study in adolescents and young adults. Eur Child Adolesc Psychiatry 17: 274-282.

Eldreth DA, Matochik A, Cadet JL, Bolla KI (2004). Abnormal brain activity in prefrontal brain regions in abstinent marijuana users. Neuroimage 23: 914920.

Filbey FM, Dunlop J, Myers US (2013). Neural effects of positive and negative incentives during marijuana withdrawal. PLoS One 8(5):e61470. doi: (10.1371/journal.pone.0061470.

Fox MD, Raichle ME (2007). Spontaneous fluctuations in brain activity observed with functional magnetic resonance imaging. Nat Rev Neurosci 8: 700711.

Gruber SA, Rogowska J, Yurgelun-Todd DA (2009). Altered affective response in marijuana smokers: An FMRI study. Drug Alcohol Depend 105: 139-153. Haber SN (2003). The primate basal ganglia: parallel and integrative networks. $J$ Chem Neuroanat 26: 317-330.

Habib M (2004). Athymhormia and disorders of motivation in basal ganglia disease. $J$ Neuropsychiatry Clin Neurosci 16: 509-524.

Harrison BJ, Soriano-Mas C, Pujol J, Ortiz H, López-Solà, Hernández-Ribas R et al (2009). Altered corticostriatal functional connectivity in obsessive-compulsive disorder. Arch Gen Psychiatry 66: 1189-1200.

Haxby JV, Hoffman EA, Gobbini MI (2002). Human neural systems for face recognition and social communication. Biol Psychiatry 51: 59-67. 
Herkenham M, Lynn AB, Little MD, Johnson MR, Melvin LS, De Costa BR et al (1990). Cannabinoid receptor localization in brain. Proc. Natl. Acad. Sci 87: 1932-1936.

Hester R, Nestor L, Garavan H (2009). Impaired error awareness and anterior cingulate cortex hypoactivity in chronic cannabis users.

Neuropsychopharmacology 34: 2450-2458.

Hindocha C, Wollenberg O, Carter Leno V, Alvarez BO, Curran HV, Freeman TP (2014). Emotional processing deficits in chronic cannabis use: A replication and extension. J Psychopharmacol 28: 466-471.

Horvitz JC (2000). Mesolimbocortical and nigrostriatal dopamine responses to salient non-reward events. Neuroscience 96: 651-656.

Jager G, Block RI, Luijten M, Ramsey NF (2013). Tentative evidence for striatal hyperactivity in adolescent cannabis-using boys: a cross-sectional multicenter fMRI study. J Psychoactive Drugs 45: 156-167.

King GR, Ernst T, Deng W, Stenger A, Gonzales RMK, Nakama H, Chang L (2011). Altered brain activation during visuomotor integration in chronic active cannabis users: Relationship to cortisol levels. The Journal of Neuroscience 31: 17923-17931.

Lang PJ, Bradley MM, Cuthbert BN (1995). International Affective Picture System (IAPS): Technical manual and affective ratings. Gainesville: University of Florida, Center for Research in Psychophysiology.

Laplane D, Dubois MD (2001). Auto-activation deficit: A basal-ganglia related syndrome. Mov Disord 16: 810-814.

This article is protected by copyright. All rights reserved. 
Leopold DA, Maier A (2013). Ongoing physiological processes in the cerebral cortex. Neuroimage 62: 2190-2200.

Marchand WR (2010). Cortico-basal ganglia circuitry: a review of key research and implications for functional connectivity studies of mood and anxiety disorders. Brain Struct Funct 215: 73-96.

Martin-Soelch C, Kobel M, Stoecklin M, Michael T, Weber S, Krebs B et al $=$ (2009). Reduced response to reward in smokers and cannabis users. Neuropsychobiology 60: 94-103.

McLaughlin RJ, Hill MN, Gorzalka BB (2014). A critical role for prefrontocortical endocannabinoid signaling in the regulation of stress and emotional behavior. Neurosci Biobehav Rev 42: 116-131.

Nestor L, Hester R, Garavan H (2010). Increased ventral striatal BOLD activity during non-drug reward anticipation in cannabis users. Neuroimage 49: 11331143.

Parvaz MA, Alia-Klein N, Woicik PA, Volkow ND, Goldstein RZ (2011). Neuroimaging for drug addiction and related behaviors. Rev Neurosci 22:609-<smiles>C1=Cc2cccc1c2</smiles>

Patton GC, Coffey C, Carlin JB, Degenhardt L, Lynskey M, Hall W (2002). Cannabis use and mental health in young people: cohort study. Br Med J 325: 1195 1198.

Paus T (2001). Primate anterior cingulate cortex: where motor control, drive and cognition interface. Nat Rev Neurosci 2: 417-424. 
Platt B, Kamboj S, Morgan CJ, Curran HV (2010). Processing dynamic facial affect in frequent cannabis-users: evidence of deficits in the speed of identifying emotional expressions. Drug Alcohol Depend 112: 27-32.

Pujol J, Blanco-Hinojo L, Batalla A, López-Solà M, Harrison BJ, Soriano-Mas C et al (2014a). Functional connectivity alterations in brain networks relevant to self-awareness in chronic cannabis users. J Psychiatr Res 51: $68-78$

Pujol J, Macià D, Garcia-Fontanals A, Blanco-Hinojo L, López-Solà M, GarciaBlanco S et al (2014b). The contribution of sensory system functional connectivity reduction to clinical pain in fibromyalgia. Pain 155: 1492-1503.

Reilly D, Didcott P, Swift W, Hall W (1998). Long-term cannabis use: characteristics of users in an Australian rural area. Addiction 93: 837-846.

Seger CA (2013). The visual corticostriatal loop through the tail of the caudate: circuitry and function. Front Syst Neurosci 7:104.

Shulz JM, Redgrave P, Carsten M, Aertsen A, Clements KM, Wickens JR et al (2009). Short-latency activation of striatal spiny neurons via subcortical visual pathways. J Neurosci 29: 6336-6347.

Skosnik PD, Krishnan GP, D’Souza DC, Hetrick WP, O’Donnell BF (2014). Disrupted gamma-band neural oscillations during coherent motion perception in heavy cannabis users. Neuropsychopharmacology 39: 30873099. 
Solowij N, Stephens RS, Roffman RA, Babor T, Kadden R, Miller M et al (2002). Cognitive functioning of long-term heavy cannabis users seeking treatment. JAMA 287: 1123-1131.

Somaini L, Manfredi M, Amore M, Zaimovic A, Raggi MA, Leonardi C et al (2012). Psychobiological responses to unpleasant emotions in cannabis users. Eur Arch Psychiatry Clin Neurosci 262: 47-57.

Thames AD, Foley JM, Wright MJ, Panos SE, Ettenhofer M, Ramezani A et al (2012). Basal ganglia structures differentially contribute to verbal fluency: Evidence from Human Immunodeficiency Virus (HIV)-infected adults. Neuropsychologia 50: 390-395.

Torrens M, Serrano D, Astals M, Pérez-Domínguez G, Martín-Santos R (2004). Diagnosing comorbid psychiatric disorders in substance abusers: validity of the Spanish versions of the psychiatric research interview for substance and mental disorders and the structured clinical interview for DSM-IV. Am J Psychiatry 16: 1231-1237.

Van Dijk KR, Sabuncu MR, Buckner RL (2012). The influence of head motion on intrinsic functional connectivity MRI. Neuroimage 59: 431-438.

van Hell HH, Vink M, Ossewaarde L, Jager G, Kahn R, Ramsey NF (2010). Chronic effects of cannabis use on the human reward system: An fMRI study. Euro Neuropsychopharmacol 20: 153-163.

Volkow ND, Wang GJ, Telang F, Fowler JS, Alexoff D, Logan J et al (2014). Decreased dopamine brain reactivity in marijuana abusers is associated with negative emotionality and addiction severity. Proc Natl Acad Sci U S A 
111(30):E3149-E3156. e-pub ahead of print 14 July 2014. doi:

10.1073/pnas.1411228111.

Wadsworth EJ, Moss SC, Simpson SA, Smith AP (2006). Cannabis use, cognitive performance and mood in a sample of workers. J Psychopharmacol 20: 14-23.

Webster KE (1975). Structure and function of the basal ganglia: a non-clinical view.

Proc R Soc Med 68: 203-210.

Wesley MJ, Hanlon CA, Porrino LJ (2011). Poor decision-making by chronic marijuana users is associated with decreased functional responsiveness to negative consequences. Psychiatry Res 191: 51-59.

Wilson CJ (2014). The sensory striatum. Neuron 83: 999-1001.

Yin HH, Knowlton BJ (2006). The role of the basal ganglia in habit formation. Nat Rev Neurosci 7: 464-476.
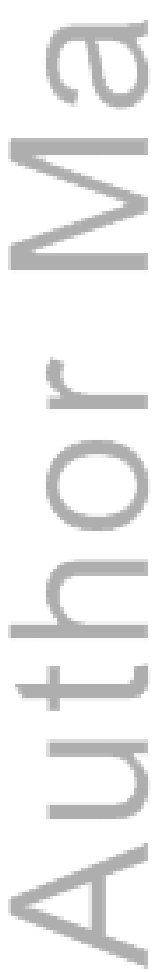

This article is protected by copyright. All rights reserved. 


\section{Figure Legends}

Figure 1. Between-group differences in the basal ganglia functional connectivity maps. Compared with control subjects, cannabis users (CU) showed a significant reduction of the positive correlation between several striatal seed regions and the ACC/medial frontal cortex (top panels), and a significant reduction of the negative correlation between several striatal regions and the fusiform gyrus (bottom panels). Result overlap across different striatal maps are illustrated in A and B. Right side of the figure corresponds to the right hemisphere for axial views. L, left hemisphere; R, right hemisphere; DC, dorsal caudate; VC, ventral caudate; VP, ventral putamen.

\section{Figure 2. Between-group differences in the ACC and fusiform gyri} functional connectivity maps. Cannabis users (CU) showed a significant reduction of the positive correlation between the anterior cingulate cortex (ACC) seed region and the basal ganglia, and a significant reduction of the negative correlation between fusiform gyri and the basal ganglia. Right side of the figure corresponds to the right hemisphere for axial and coronal views. L, left hemisphere; R, right hemisphere.

Figure 3. Plots of the correlations between measurements of cannabis use and functional connectivity. Longer history of cannabis use was associated with lower functional connectivity between the left dorsal caudate and the ACC/medial frontal cortex (top panel; peak correlation at MNI coordinates $\mathrm{x}=0, \mathrm{y}=34, \mathrm{z}=32$; $\mathrm{T}=3.4$ ), whereas greater amount of cannabinoid metabolites in urine was associated 
with higher functional connectivity (decreased anticorrelation) between the right ventral putamen and the left fusiform gyrus (bottom panel; peak correlation at MNI $x=-36, y=-42, z=-20 ; T=3.9)$. The boxplots illustrate reference functional connectivity values in the control group. The right hemisphere corresponds to the right side of the axial view. DC, dorsal caudate; VP, ventral putamen; A.u., arbitrary units.

\section{Figure 4. Correlation of arousal ratings with functional connectivity.}

Primary analysis (top row): In cannabis users, lower levels of arousal were associated with weaker positive correlation between basal ganglia and the default-mode network (top left), and with weaker negative correlation between the basal ganglia and the sensorimotor cortex (top right). In the extended analysis (bottom row): lower arousal correlated with weaker connectivity between basal ganglia and anterior cingulate cortex (ACC) (bottom left), and with weaker negative correlation between the basal ganglia and the fusiform gyrus (bottom right). Right side of the figure corresponds to the right hemisphere for both axial and coronal views. VC, ventral caudate; DC, dorsal caudate.

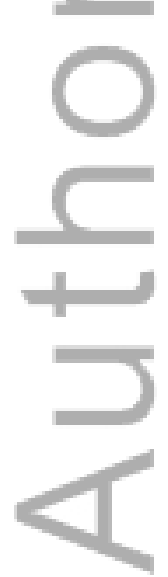

This article is protected by copyright. All rights reserved. 
Table 1. Cannabis use and behavioral tests

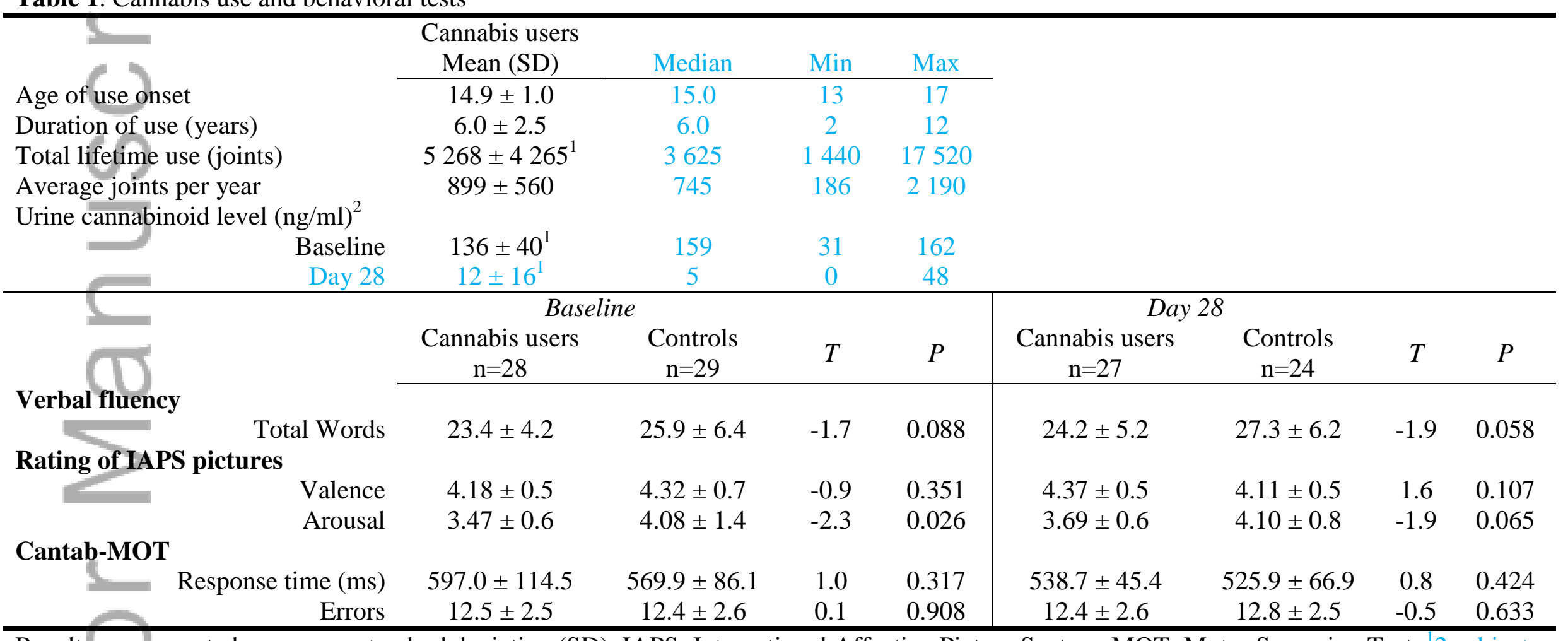

Results are presented as mean \pm standard deviation (SD). IAPS: International Affective Picture System. MOT: Motor Screening Test. ${ }^{1} 2$ subjects

were outliers; ${ }^{2} \mathrm{n}=22$. 


\section{Correlation decrease in $\mathbf{C U}$}
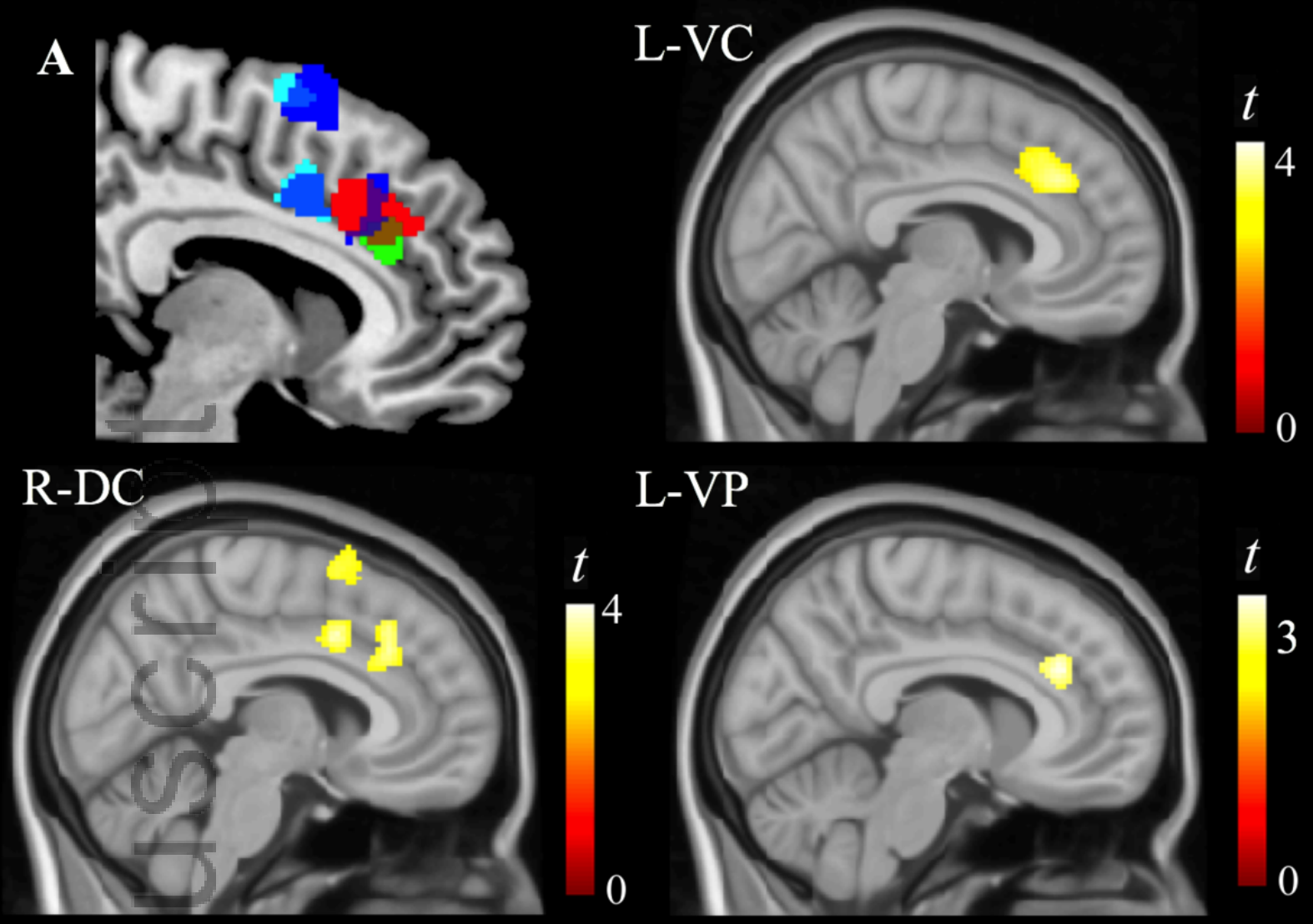

Anticorrelation decrease in CU
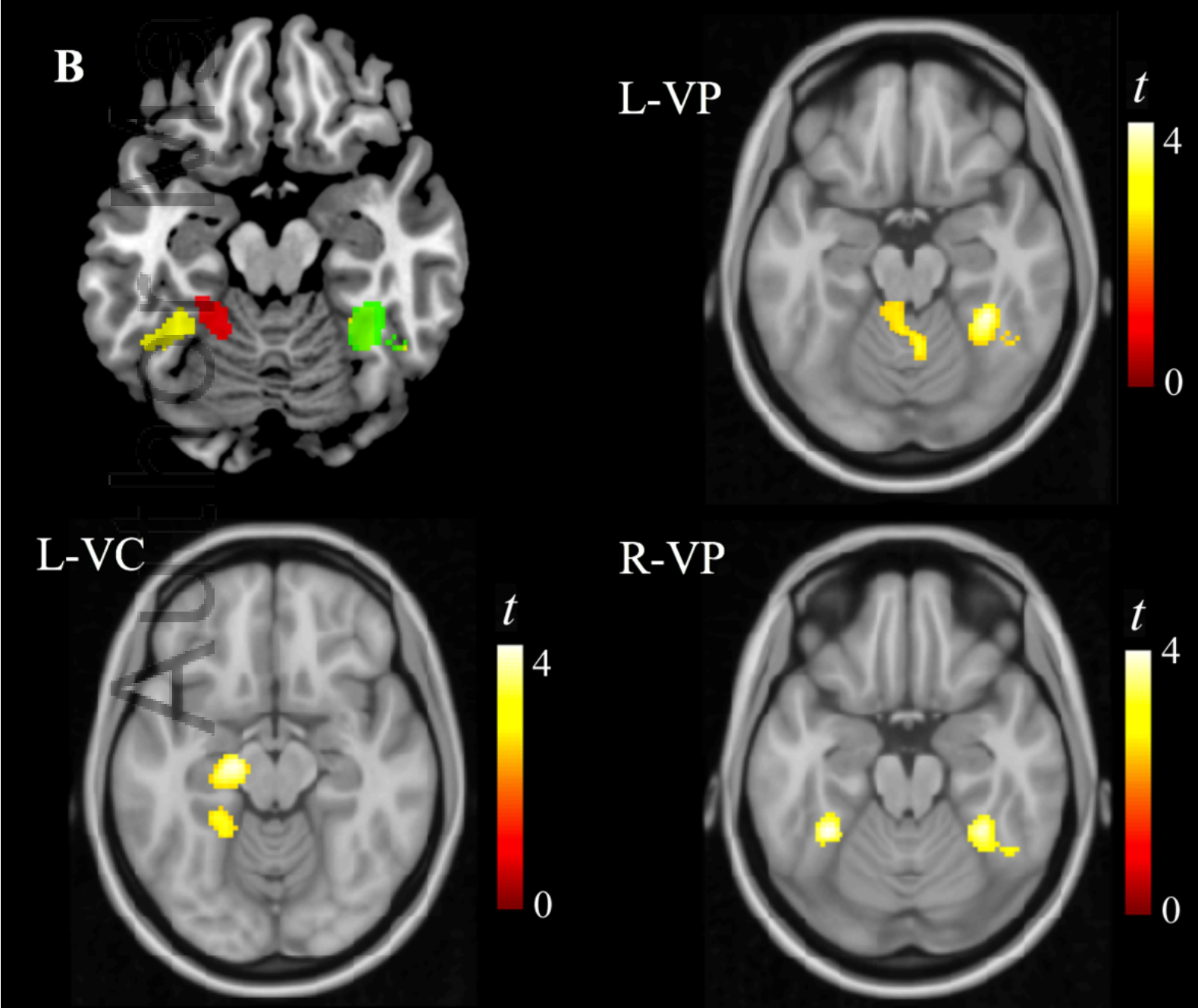


\section{Correlation decrease in $\mathbf{C U}$}
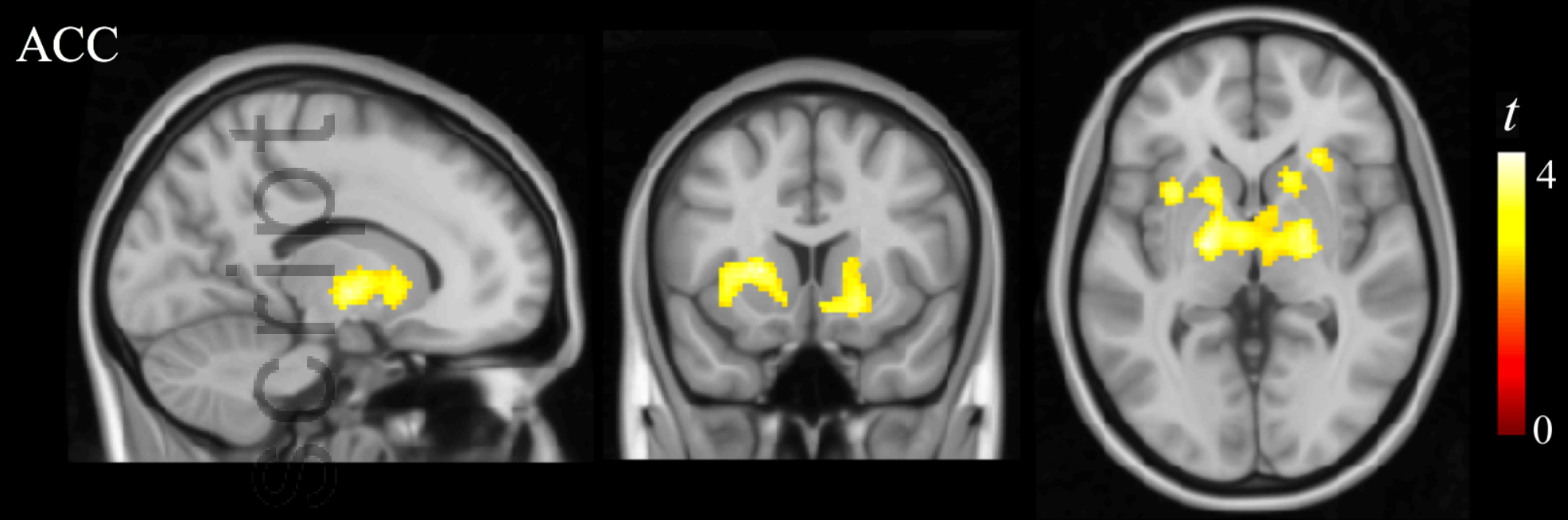

Anticorrelation decrease in $\mathbf{C U}$

L Fusiform

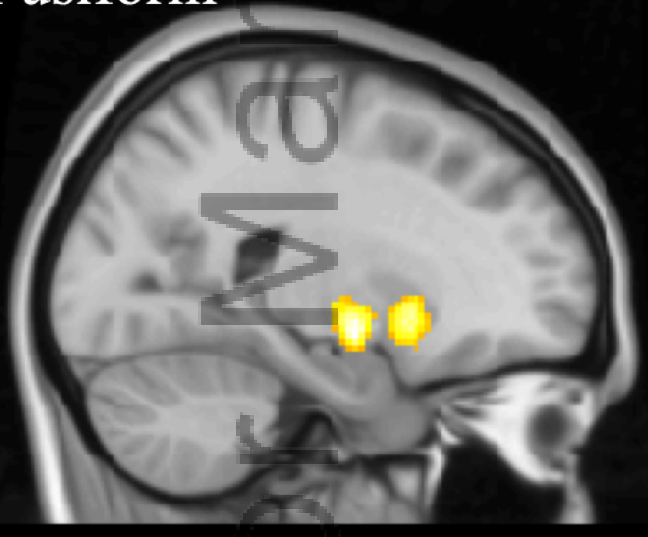

R Fusiform

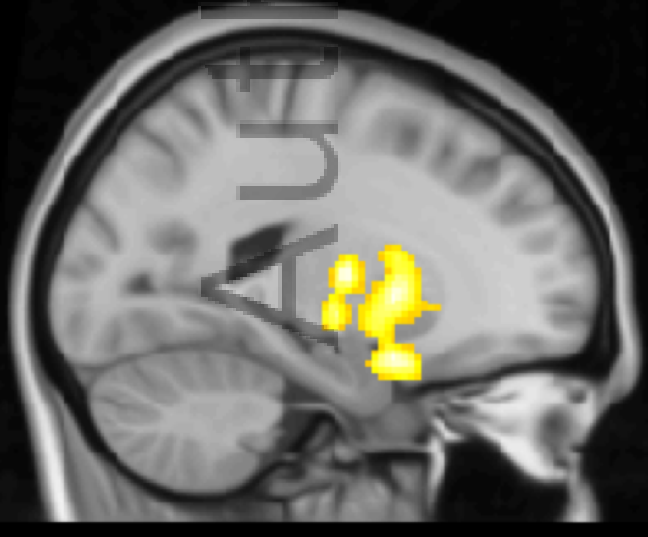

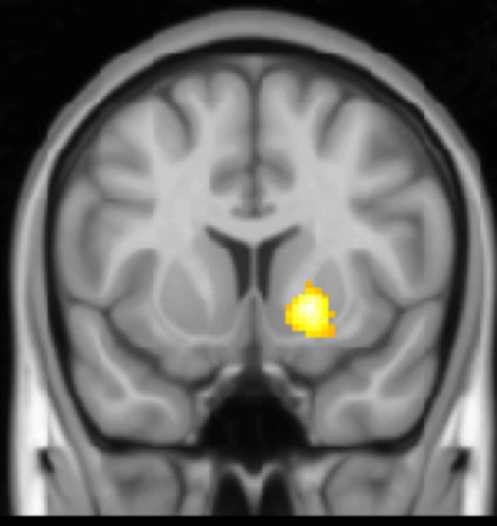
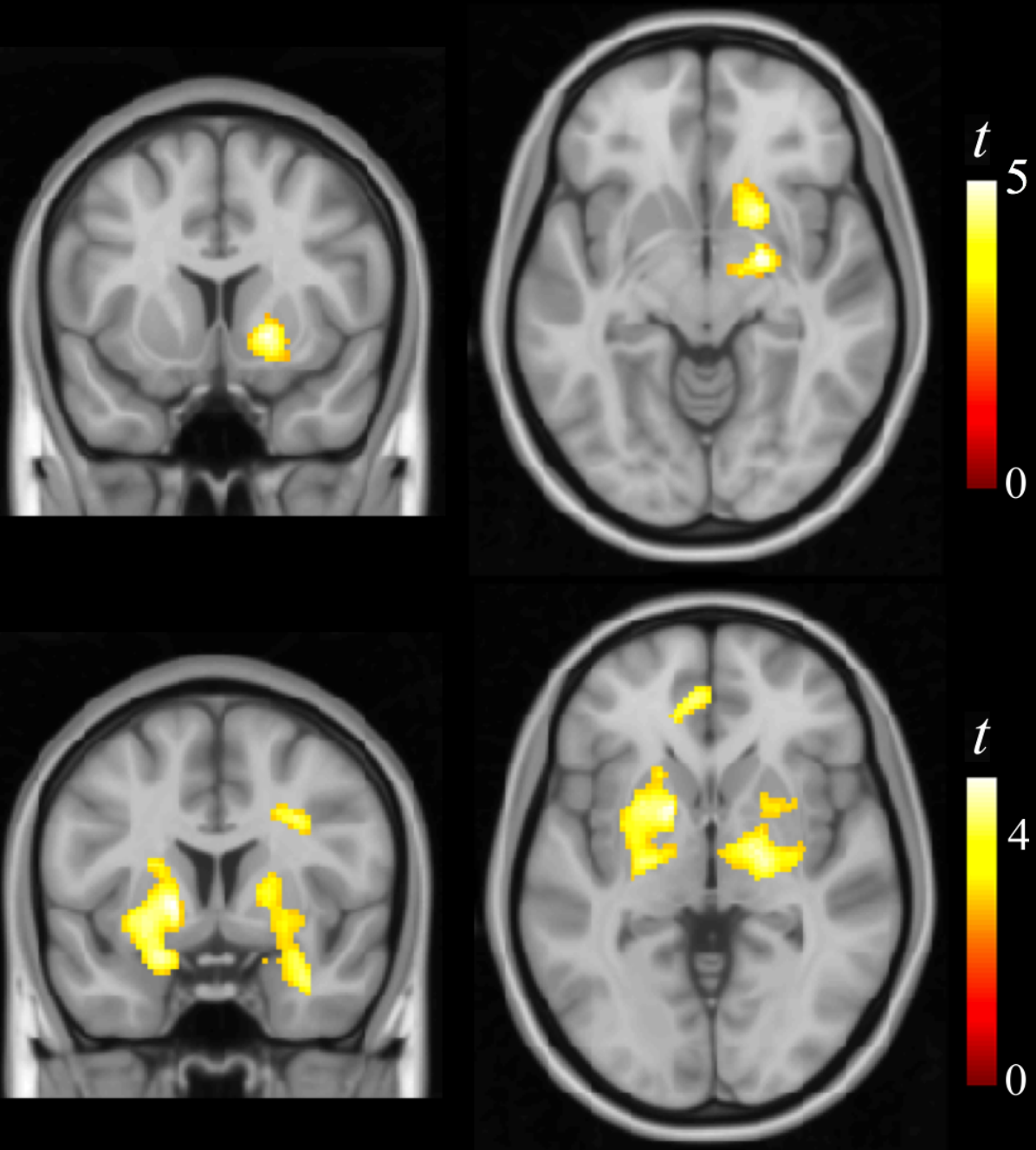

ADB_12370_F2.tif

This article is protected by copyright. All rights reserved. 


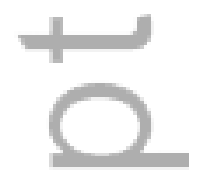

in

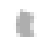

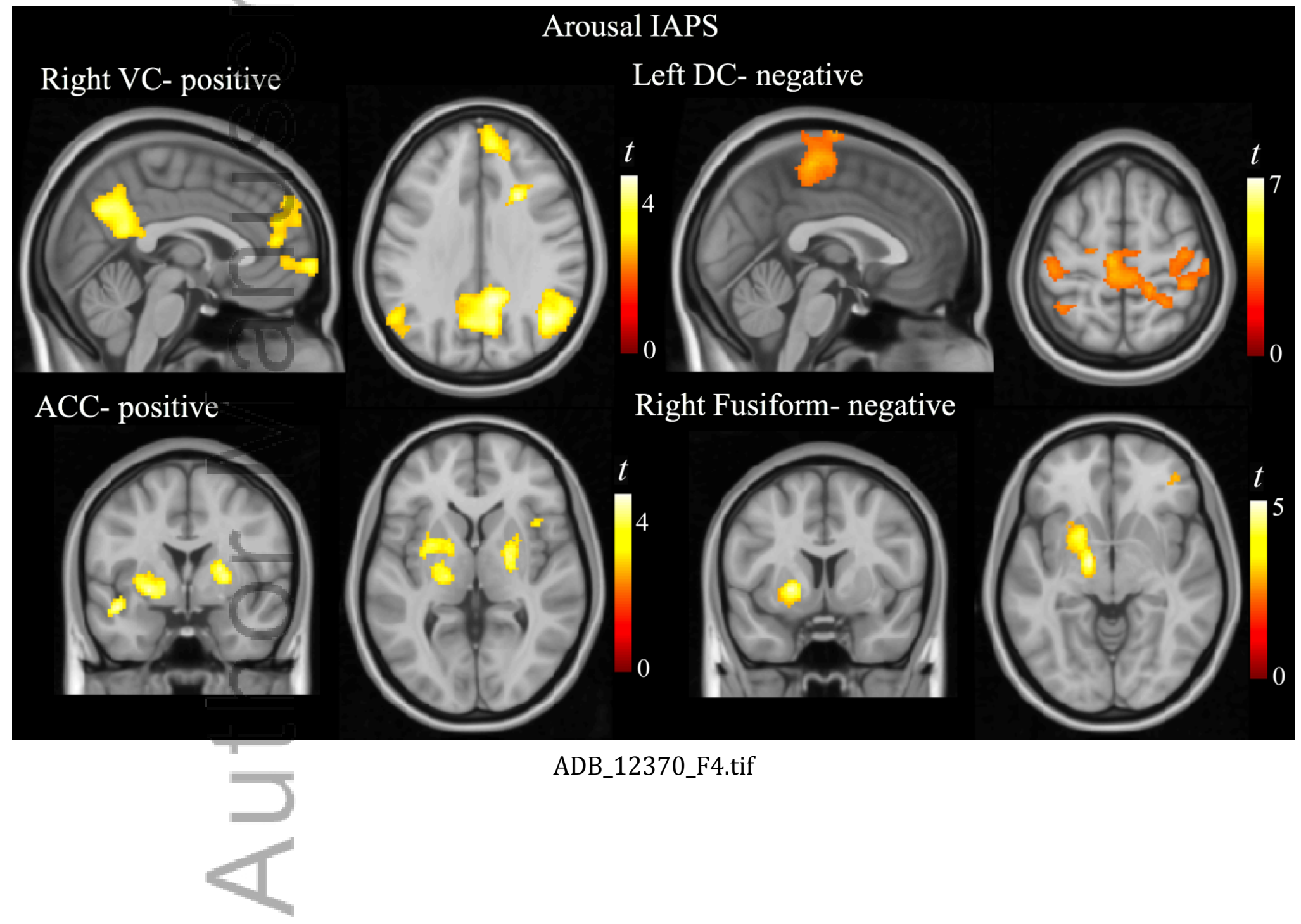

This article is protected by copyright. All rights reserved. 


\section{University Library}

\section{- M M N E R VA A gateway to Melbourne's research publications}

Minerva Access is the Institutional Repository of The University of Melbourne

Author/s:

Blanco-Hinojo, L;Pujol, J;Harrison, BJ;Macia, D;Batalla, A;Nogue, S;Torrens, M;Farre, M;Deus, J;Martin-Santos, R

Title:

Attenuated frontal and sensory inputs to the basal ganglia in cannabis users

Date:

2017-07-01

Citation:

Blanco-Hinojo, L., Pujol, J., Harrison, B. J., Macia, D., Batalla, A., Nogue, S., Torrens, M., Farre, M., Deus, J. \& Martin-Santos, R. (2017). Attenuated frontal and sensory inputs to the basal ganglia in cannabis users. ADDICTION BIOLOGY, 22 (4), pp.1036-1047. https:// doi.org/10.1111/adb.12370.

Persistent Link:

http://hdl.handle.net/11343/291018 